\title{
CT imaging history for patients presenting to the ED with renal colic--evidence from a multi-hospital database
}

\author{
Emily Schmid ${ }^{1}$, Kimberly Leeson ${ }^{1}$, K. Tom Xu², Peter Richman ${ }^{1,3^{*}}$, Crystal Nwosu ${ }^{1}$ and Lynn Carrasco ${ }^{1}$
}

\begin{abstract}
Background: Patients with renal colic have a $7 \%$ chance of annual recurrence. Previous studies evaluating cumulative Abbreviations: computed tomography $(\mathrm{CT})$ exposure for renal colic patients were typically from single centers.

Methods: This was an observational cohort study. Inner-city ED patients with a final diagnosis of renal colic were prospectively identified (1/10/16-10/16/16). Authors conducted structured electronic record reviews from a 6-hospital system encompassing over 192,000 annual ED visits. Categorical data analyzed by chi-square; continuous data by ttests. Primary outcome measure was the proportion of study group patients with prior history $C T$ abdomen/pelvis $C T$.

Results: Two hundred thirteen patients in the study group; 59\% male, age 38+/- 10 years, 67\% Hispanic, 62\% prior stone history, flank pain (78\%), dysuria (22\%), UA (+) blood (75\%). 60\% (95\% Cl=53-66\%) of patients received an EDCV CT; hydronephrosis seen in 55\% (95\% Cl=46-63\%), stone in 90\%(95\% Cl=83-94\%). No significant differences observed in the proportion of EDCV patients who received $C T$ with respect to: female vs. male ( $62 \%$ vs. $56 \% ; p=0.4$ ), mean age (37+/- 9 years vs. 39+/- 11 years; $p=0.2)$, and Hispanic vs. non-Hispanic white (63\% vs.63\%; $p=0.96)$. Patients with a prior stone history were more likely than those with no history to receive an EDCV CT (88\% vs. 16\%; $p<0.001) .118$ (55\%; 95\% Cl=49-62\%) of patients had at least one prior CT, 46 (22\%; 95\% Cl = 16-28\%) had $\geq 3$ prior CTs; 29 (14\%; 95\% Cl=10-19\%), $\geq 10$ prior CTs. Patients who did not receive an EDCV CT had a significantly higher mean prior number of $C T s$ than those who had EDCV $\subset T(5.1+/-7.7$ vs $2.2+/-4.9 ; p<0.001)$. Patients with prior stone were more likely to receive only U/S during EDCV (33\% vs. 15\%; $p=0.003)$.

Conclusions: Within our EDCV cohort of renal colic patients, 55\% had at least one prior $C T$. The mean number of prior CTs was lower for patients receiving CT on EDCV, and Ultrasound (US) alone was used more often in patients with prior stone history vs. those with no prior history.
\end{abstract}

Keywords: Renal colic, Repeat imaging, CT scan

\section{Background}

Numerous investigators have raised concerns regarding the potential relationship between ionizing radiation dose from medical imaging and risk of human malignancy [1-5]. Depending on the area imaged, a single CT scan is estimated to increase the lifetime risk of cancer by as much as 1 in 2000 [3]. Despite such risk, the use

\footnotetext{
* Correspondence: PRichmanMDMBA@gmail.com

'Department of Emergency Medicine, CHRISTUS Health/Texas A\&M School of Medicine, Corpus Christi, TX, USA

${ }^{3}$ CHRISTUS HEALTH/Texas A\&M Residency in Emergency Medicine, 600

Elizabeth Street, Corpus Christi, TX 78404, USA

Full list of author information is available at the end of the article
}

of CT has grown significantly over the past 20 years to evaluate for pulmonary embolism and a variety of intra-abdominal conditions. The average growth rate of CT utilization for chest pain complaints alone was 28.1\% per year between 2001 and 2007 [6].

Several publications in the mid-1990s touted the use of CT to accurately identify the presence of nephrolithiasis and alternative conditions in ED patients with suspected renal colic. Within just a few years, unenhanced CT of the abdomen and pelvis supplanted intravenous pyelography (IVP) as the diagnostic study of choice for evaluating patients with suspected renal colic.

(c) The Author(s). 2019 Open Access This article is distributed under the terms of the Creative Commons Attribution 4.0 International License (http://creativecommons.org/licenses/by/4.0/), which permits unrestricted use, distribution, and reproduction in any medium, provided you give appropriate credit to the original author(s) and the source, provide a link to the Creative Commons license, and indicate if changes were made. The Creative Commons Public Domain Dedication waiver (http://creativecommons.org/publicdomain/zero/1.0/) applies to the data made available in this article, unless otherwise stated. 
[7] Similar to the observed trends for chest imaging, CT utilization for the evaluation of patients with flank pain grew exponentially $[7,8]$. Westphalen et al. found that from 1996 to 2007 there was a 10-fold increase in CT scan use for patients with suspected kidney stone, but the authors found no associated change in the proportion of diagnosis of kidney stone, diagnosis of significant alternate diagnoses, or admission to the hospital [7]. A similar study conducted from 2000 to 2008 revealed a significant increase in computerized tomography use, a significant decrease in $\mathrm{x}$-ray use, historically the preferred imaging study for this condition, while ultrasound use remained stable. They also found that despite the increased use of CT scan, the proportion of patients diagnosed with kidney stone did not increase [8].

The growing use of CT scan as the diagnostic modality of choice in renal colic patients is particularly concerning because of the recurrent nature of the condition. The average patient with renal colic has a 7\% chance of recurrence per year and a $50 \%$ chance of a recurrence within 10 years [9]. A single center, retrospective chart review including 356 patient encounters over a 10-month period by Broder et al. found that $79 \%$ of patients presenting to emergency departments with recurrent symptoms suggesting renal colic underwent at least 2 CT scans [10]. Katz et al. estimated that a typical single detector unenhanced CT and single multi-detector unenhanced CT of the abdomen and pelvis exposes a patient to $6.5 \mathrm{mSv}$ and $8.5 \mathrm{mSv}$ respectively [5]. Unfortunately, within their single-center cohort of 4562 patients undergoing repeat CT scan for suspected renal colic, they found patients with three or more unenhanced CT scans of the abdomen and pelvis were exposed to ionizing radiation doses ranging from 20 to $154 \mathrm{mSv}$. Such exposure falls within a range that Brenner et al. found suggestive of significantly increased cancer risks $(10-50 \mathrm{mSv}$ for an acute exposure and $50-100 \mathrm{mSv}$ for a protracted exposure) [4].

Prior studies related to CT scan use in renal colic have been mostly small, single center studies or have relied on national, administrative databases [5, 7-15]. We are unaware of prospective emergency department-based studies, to date, that evaluated repeated patient exposure for renal colic imaging within a multi-center database. The objective of our study was to evaluate whether our patients had been exposed to multiple CTs for the evaluation of renal colic within a regional hospital system and to identify patient characteristics that were associated with $\mathrm{CT}$ use during the current $\mathrm{ED}$ visit.

\section{Methods}

\section{Study design}

This was a multi-center prospective observational cohort study of inner-city patients designed to compare characteristics and imaging history from a six-hospital database for a cohort of patients diagnosed with renal colic during their current ED visit (EDCV). Subjects were verbally consented for participation.

\section{Setting}

Patients were enrolled that presented to CHRISTUS Spohn Memorial hospital emergency department (Corpus Christi, Texas). The facility is a teaching affiliate of the Texas A\&M Health Science Center, a level-two trauma center, and serves an inner-city population. The annual Emergency Department census is 45,000 patients. The study was approved by the CHRISTUS Spohn Institutional Review Board prior to initiation of patient enrollment.

\section{Population}

Adult patients greater than 18 years of age that received a diagnosis of renal colic at their current ED visit were eligible for enrollment. The CHRISTUS Spohn Institutional Review Board provided a waiver of consent as all data was available in the medical record. Patients less than 18 years of age and pregnant patients were excluded from the study.

\section{Study protocol}

CHRISTUS Spohn Memorial emergency physicians with the aid of trained research associates prospectively identified the patient cohort over a 10-month period from January 10, 2016 to October 16, 2016 at time of care. Demographic and historical features were recorded on a data collection form. To determine prior imaging exposure and CT results for patients, the study authors conducted structured chart reviews of a shared electronic medical record (EMR) for a 6-hospital system, including 192,073 annual ED visits (70.6\% of all ED visits within a twelve-county region). The study also evaluated the method by which the patient was diagnosed with renal colic at the current ED visit, including laboratory and imaging results.

\section{Statistical methods}

Categorical data was analyzed by chi square and 95\% confidence intervals were calculated. Continuous data was analyzed by t-tests. All tests were two-tailed; alpha set at 0.05 . The primary outcome parameter was to observe the proportion of patients with renal colic who had a history of multiple CTs for renal colic within our system. Secondary outcomes included identifying patients' characteristics that were associated recurrent CT use, CT use for the current visit, and ultrasound use for the current visit. 


\section{Results}

Patient characteristics are shown in Table 1. Of the 213 patients included in the final study group, 59\% were male and $67 \%$ were Hispanic. The mean age was $38+/-$ 11 years. Sixty-two percent of patients reported a prior history of kidney stones. The most common presenting symptom was flank pain and hematuria was detected by urinalysis in $76 \%$ of patients (Table 2 ).

Table 3 reveals that $60 \%(95 \% \mathrm{CI}=53-66 \%)$ of patients received a $\mathrm{CT}$ scan at the ED current visit and $30 \%$ of patients underwent US. Only $4 \%$ of patients underwent both a CT and an US and $14 \%$ of patients had no imaging performed at their current visit. Hydronephrosis was present on $55 \%$ (95\% CI $=46-63 \%)$ of CTs and $28 \%$ (95\% CI $=19-40 \%$ ) of ultrasounds performed during the current visit. A renal stone was identified on $90 \%$ (95\% $\mathrm{CI}=83-94 \%)$ of CTs and 38\% (95\% CI $=45-74 \%)$ of ultrasounds performed in those cases.

No significant differences were observed in the proportion of patients for the current ED visit who received CT with respect to: female vs. male ( $62 \%$ vs. $56 \% ; p=$ $0.4)$, mean age $(37+/-9$ years vs. $39+/-11$ years; $p=0.2)$, and Hispanic vs. non-Hispanic white (63\% vs.63\%; $p=0.96)$. Patients with a prior stone history were more likely than those with no history to receive an EDCV CT $(88 \%$ vs. $16 \% ; p<0.001)$. Patients with a urinalysis positive for blood were no more likely to undergo $\mathrm{CT}$ than patients with no blood on urinalysis (61\% vs. $60 \% ; \mathrm{p}=0.4)$.

Table 4 shows patients' prior CT histories. Out of the 213 patients, 118 (55\%; 95\% CI $=49-62 \%)$ had at least one prior CT, 46 (22\%; 95\% CI $=16-28 \%)$ had $\geq 3$ prior CTs, and 29 patients (14\%; 95\% CI $=10-19 \%)$ had $\geq 10$ prior CTs. Patients who did not receive a CT at the current visit had a significantly higher mean prior number of CTs than those who had current visit CT $(5.1+/-$ 7.7 vs $2.2+/-4.9 ; p<0.001)$. Patients who received an US at their current visit also had a significantly higher mean number of prior CTs $(4.9+/-7.6$ vs $2.8+/-5.6$; $\mathrm{p}<$ $0.001)$. Characteristics associated with positive prior CT history included: female gender ( $42 \%$ vs. $28 \% ; p=0.02)$, flank pain $(40 \%$ vs. $13 \% ; \mathrm{p}<0.001)$, and prior renal stone by history ( $48 \%$ vs. $10 \%$; $\mathrm{p}<0.001$ ).

Patients with a reported prior stone were more likely to receive a renal ultrasound as the only imaging study during the EDCV (33\% vs. $15 \%$; $p=0.003)$. Patients with

Table 1 Presenting signs, symptoms, and laboratory findings

\begin{tabular}{ll}
\hline Flank pain & $78 \%$ \\
Abdominal pain & $50 \%$ \\
Nausea and/or vomiting & $62 \%$ \\
Dysuria & $22 \%$ \\
Blood on urinalysis & $76 \%$ \\
\hline
\end{tabular}

Table 2 Patient prior CT scans of the abdomen/pelvis

\begin{tabular}{lll}
\hline Number of prior CT scans & Frequency & Percent \\
\hline 0 & 95 & 45 \\
1 & 46 & 22 \\
2 & 17 & 8 \\
3 & 11 & 5 \\
4 & 6 & 3 \\
5 & 2 & 1 \\
6 & 1 & 0.5 \\
7 & 6 & 3 \\
12 & 1 & 0.5 \\
13 & 12 & 6 \\
14 & 3 & 1 \\
20 & 1 & 0.5 \\
23 & 2 & 1 \\
24 & 5 & 2 \\
25 & 4 & 2 \\
26 & 1 & 0.5 \\
\hline
\end{tabular}

a history of one or more CT scans were also more likely to undergo only an ultrasound compared to those with no prior CT (35\% vs. $22 \%$; $p=0.046)$. For patients who received only an US at their current visit, there were no significant differences observed with respect to female vs. male $(26 \%$ vs. $26 \% ; p=1.0)$, mean age $(37+/-9$ vs. 38 $+/-11)$, or Hispanic vs. non-Hispanic white $(23 \%$ vs. $25 \% ; p=0.4$ ).

\section{Discussion}

Unenhanced CT is a rapid and highly accurate imaging modality with reported specificity and sensitivity between 91 and 100\% for diagnosing nephrolithiasis in patients presenting with flank pain $[12,13]$. In theory, physicians and their patients benefit from CT use as it may also identify alternative significant diagnoses. However, somewhat surprisingly, the exponential increase in the use of CT scan for evaluation of flank pain has not resulted in significant improvements such as finding alternate diagnoses, better diagnosis of kidney stone, changing rates of admissions to the hospital, and/or identifying those in need of immediate urologic intervention $[7,14]$.

Table 3 Current visit imaging

\begin{tabular}{ll}
\hline CT & $60 \%$ \\
US & $30 \%$ \\
US only & $27 \%$ \\
CT only & $56 \%$ \\
No imaging & $14 \%$ \\
CT and US & $4 \%$ \\
\hline
\end{tabular}


Table 4 Patient prior CT scans of the abdomen/pelvis

\begin{tabular}{lll}
\hline Number of prior CT scans & Frequency & Percent \\
\hline 0 & 95 & 45 \\
1 & 46 & 22 \\
2 & 17 & 8 \\
3 & 11 & 5 \\
4 & 6 & 3 \\
5 & 2 & 1 \\
6 & 1 & 0.5 \\
7 & 6 & 3 \\
12 & 1 & 0.5 \\
13 & 12 & 6 \\
14 & 3 & 1 \\
20 & 1 & 0.5 \\
23 & 2 & 1 \\
24 & 5 & 2 \\
25 & 4 & 2 \\
26 & 1 & 0.5 \\
\hline
\end{tabular}

Several recent studies propose that renal ultrasound should be used as the first or even only diagnostic study when evaluating renal colic. The advantages of ultrasound include a low cost and widespread availability [16]. Investigators have reported that utilizing ultrasound as the initial diagnostic study of choice for renal colic is associated with lower cumulative radiation exposure without significant differences in complications, serious adverse events, pain scores, return emergency department visits, or hospitalizations [15]. In addition, studies comparing $\mathrm{CT}$ and US have shown a specificity of $95-100 \%$ and sensitivity between 61 and $93 \%$ for diagnosing ureterolithisis with US. These results suggest that US may be the ideal screening test, while CT is be reserved for situations when US is unavailable or non-diagnostic $[12,13]$.

Another proposed benefit to using CT is the ability to accurately measure stone size and degree of urinary obstruction. CT can also provide information about the need for further urologic intervention such as stenting [9]. However, authors of a recent study observed that the vast majority of patients receiving a CT scan as part of their work-up for renal colic did not require admission or immediate intervention for their kidney stone. Thus, there is a large population of low risk patients with suspected renal stones that could safely be managed with either ultrasound alone, expectant management or delayed CT [14]. Unfortunately, despite evidence that ultrasound is a cost-effective and useful alternative without inherent radiation risks, CT utilization continues to be the prevalent modality for diagnosis of ureterolithiasis and renal colic in the ED $[7,8,10,14]$.
We believe that our study is novel for utilizing a multi-hospital database across a geographic region to determine the prior imaging exposure of our ED patients. While surely not inclusive of all prior studies that might have been obtained at free-standing and non-system hospitals, our hospital system captures the majority of ED visits in a 12-county region. Further, we suggest that our study adds to the current understanding of imaging patterns for ED patients as we examined patient characteristics and clinical history that was associated with CT use both at the current visits and past visits respectively.

Our study demonstrated a lower rate of CT scanning in this population than that which has been reported in other similar studies. $(9,12-15)$ We observed that during the current visit, $60 \%$ of all patients presenting with suspected renal colic underwent CT imaging as part of their diagnostic work-up. Within our cohort, patients reporting a history of kidney stones were much more likely to undergo a CT scan on the current visit than those with no reported kidney stone history ( $88 \%$ versus $16 \% ; p<0.001)$. Disturbingly, within our multi-hospital system EMR, we found that $55 \%$ of all enrolled patients had at least one prior CT, $22 \%$ had 3 or more prior CTs, and $14 \%$ had 10 or more CTs.

On the other hand, we report findings that suggest that our physicians may have been aware of the patients' imaging history. Patients who did not receive a current $E D$ visit $C T$ had a significantly higher mean prior number of CTs than those who underwent a CT scan at the current visit $(5.1+/-7.7$ vs $2.2+/-4.9 ; \mathrm{p}<0.001)$. This notion is further supported by our observation that patients with a prior stone history were much more likely to receive only a renal US during the EDCV (33\% vs. $15 \% ; p=0.003)$.

\section{Limitations and future questions}

Our study has several limitations that warrant discussion. Our study population was predominantly Hispanic and from lower socioeconomic groups. Thus, our findings may not be generalizable to other settings. As we noted previously, our methodology likely underestimates the prior imaging exposure of our patients as we cannot account for CTs performed outside of our hospital system. Regardless of such a limitation, our records identified a large percentage of patients with a history of recurrent imaging, so the findings are, nonetheless concerning. Finally, although our data suggest some reason for hope that the practice of emergency physicians may be evolving toward primary use of ultrasound for work-up of renal colic, our practice setting may not be generalizable to others as we have a teaching environment with residents and clinical faculty providing care to patients. Future investigators should examine the evolution of imaging practice for ED renal colic evaluation 
in other types of institutions and for other patient populations.

\section{Conclusions}

Within our ED cohort of renal colic patients, 55\% had at least one prior CT. The mean number of prior CTs was lower for patients receiving $\mathrm{CT}$ on EDCV, and Ultrasound (US) alone was used more often in patients with prior stone history vs. those with no prior history.

\section{Abbreviations}

CT: Computed tomography; EDCV: Emergency department visit; IVP: Intravenous pyelography; US: Ultrasound

\section{Acknowledgements}

Not applicable.

\section{Funding}

Not applicable.

\section{Availability of data and materials}

All data generated or analyzed during this study are included in this published article.

\section{Authors' contributions}

ES as principal investigator was responsible for all aspects of this study. $\mathrm{KL}$ was a contributor in writing the manuscript. KTX performed the statistical analysis for this study. PR Made extensive contributions for conception and design of this study as well as in writing the manuscript. CN performed a major role in consenting subjects and preparing data collection. LC contributed to the design, acquiring of data, accuracy and writing of this study. All authors read and approved the final manuscript.

\section{Ethics approval and consent to participate}

CHRISTUS Health IRB reviewed and approved this study for ethics and participation of human subjects. Their approval number for this study is 2015-049.

The CHRISTUS Spohn Institutional Review Board provided a waiver of consent as all data was available in the medical record.

\section{Consent for publication}

Not applicable.

\section{Competing interests}

The authors declare that they have no competing interests.

\section{Publisher's Note}

Springer Nature remains neutral with regard to jurisdictional claims in published maps and institutional affiliations.

\section{Author details}

'Department of Emergency Medicine, CHRISTUS Health/Texas A\&M School of Medicine, Corpus Christi, TX, USA. ${ }^{2}$ Texas Tech University School of Medicine, Bullock, Lubbock, TX, USA. ${ }^{3}$ CHRISTUS HEALTH/Texas A\&M Residency in Emergency Medicine, 600 Elizabeth Street, Corpus Christi, TX 78404, USA.

Received: 19 April 2018 Accepted: 4 February 2019

Published online: 01 March 2019

\section{References}

1. De Jong PA, Mayo JR, Golmohammadi K, Nakano Y, Lequin MH, Tiddens HA, Aldrich J, Coxson HO, Sin DD. Estimation of cancer mortality associated with repetitive computed tomography scanning. Am J Respir Crit Care Med. 2006;173:199-203.

2. Brenner DJ, Elliston CD. Estimated radiation risks potentially associated with full-body CT screening. Radiology. 2004;232:735-8.

3. Radiation and your patient: a guide for medical practitioners. Ann ICRP $2001 ; 31: 5-31$
4. Brenner DJ, Doll R, Goodhead DT, Hall EJ, Land CE, Little JB, Lubin JH, Preston DL, Preston RJ, Puskin JS, Ron E, Sachs RK, Samet JM, Setlow RB, Zaider M. Cancer risks attributable to low doses of ionizing radiation: assessing what we really know. Proc Natl Acad Sci U S A. 2003;100:13761-6.

5. Katz SI, Saluaja S, Brink JA, Forman HP. Radiation dose associated with unenhanced CT for suspected renal colic: impact of repetitive studies. AJR Am J Roentgenol. 2006;186:1120-4.

6. Feng LB, Pines JM, Yusuf HR, Grose SD. U.S. trends in computed tomography use and diagnoses in emergency department visits by patients with symptoms suggestive of pulmonary embolism, 2001-2009. Acad Emerg Med. 2013;20(10):1033-40.

7. Westphalen AC, Hsia RY, Maselli JH, Wang R, Gonzales R. Radiological imaging of patients with suspected urinary tract stones: national trends, diagnoses, and predictors. Acad Emer Med. 2011;18:700-7.

8. Hyams ES, Korley FK, Pham JC, Matlaga BR. Trends in imaging use during the emergency department evaluation of flank pain. J of Urol. 2011;186(6): 2270-4

9. Prina LD, Rancatore E, Secic M, Weber RE. Comparison of stone size and response to analgesic treatment in predicting outcome of patients with renal colic. Eur J Emerg Med. 2002;9:135-9.

10. Broder J, Bowen J, Lohr J, Babcock A, Yoon J. Cumulative CT exposures in emergency department patients evaluated for suspected renal colic. J of Emerg Med. 2007;33(2):161-8.

11. Smith RC, Rosenfield AT, Choe KA, Essenmacher KR, Verga M, Glickman MG, Lange RC. Acute flank pain: comparison of non-contrast-enhanced CT and intravenous urography. Radiology. 1995;194:789-94.

12. Patlas M, Farkas A, Fisher D, Zaghal I, Hadas-Halpern I. Ultrasound vs CT for the detection of ureteric stones in patients with renal colic. Br J Radiol. 2001;74:901-4

13. Sheafor DH, Hertzberg BS, Freed KS, Carroll BA, Keogan MT, Paulson EK, DeLong DM, Nelson RC. Nonenhanced helical CT and US in the emergency evaluation of patients with renal colic: prospective comparison. Radiology. 2000;217:792-7.

14. Schoenfeld EM, Pekow PS, Shieh MS, Scaled CD Jr, Lagu T, Lindenauer PK. The diagnosis and management of patients with renal colic across a sample of US hospitals: high CT utilization despite low rates of admission and inpatient urologic intervention. PLoS One. 2017;12(1):e0169160.

15. Smith-Bindman R, Aubin C, Bailitz J, Bengiamin RN, Camargo CA Jr, Corbo J, Dean AJ, Goldstein RB, Griffey RT, Jay GD, Kang TL, Kriesel DR, Ma OJ, Mallin M, Manson W, Melnikow J, Miglioretti DL, Miller SK, Mills LD, Miner JR, Moghadassi M, Noble VE, Press GM, Stoller ML, Valencia VE, Wang J, Wang RC, Cummings SR. Ultrasonography versus computed tomography for suspected nephrolithiasis. N Engl J Med. 2014;371(12):1100-10.

16. Nicolau C, Claudon M, Derchi LE, Adam EJ, Bachmann Nielsen M, Mostbeck G, Owens CM, Nyhsen C, Yarmenitis S. Imaging patients with renal colicconsider ultrasound first. Insights Imaging. 2015;6(4):441-7.

\section{Ready to submit your research? Choose BMC and benefit from:}

- fast, convenient online submission

- thorough peer review by experienced researchers in your field

- rapid publication on acceptance

- support for research data, including large and complex data types

- gold Open Access which fosters wider collaboration and increased citations

- maximum visibility for your research: over $100 \mathrm{M}$ website views per year

At $B M C$, research is always in progress.

Learn more biomedcentral.com/submissions 